\title{
Adaptive control effect analysis with parameters not totally
}

\section{identified}

\author{
Yuliang Chen ${ }^{\text {a }}$, Haijun Li and Tao Jiang \\ Department of Ordnance Science and Technology, Naval aeronautical and astronautical University \\ Yantai, China \\ achenyuliang1024@126.com
}

Key words: adaptive control, parameter identification, one order system, final value, stability

Abstract: In view of the fact that the parameters can not be fully identified, which is frequently occurred in real adaptive parameter identification, this paper makes a deep analysis and studies the effect of the adaptive control law on the situation that parameter is not fully identified. In order to simplify the problem, this paper selects a class of first order systems with unknown parameters, the controller design and numerical simulation analysis are carried out by using the method of non adaptive control and adaptive control method, the results show that the adaptive control can replace the integral control, which can eliminate the steady-state error of the system.

\section{Introduction}

In 1980s, the development of differential geometry theory of nonlinear feedback control systems has stimulated the research interest of adaptive control for nonlinear systems. Although nonlinear differential geometry theory has many advantages, it is not long before people realize that it also has some disadvantages ${ }^{[1-5]}$. One of them is that it can not deal with the unknown parameters in the system, which makes the emergence of the first class of adaptive nonlinear control strategy ${ }^{[6-9]}$. After 30 years of development, adaptive control has made great progress both in theory and in application. But this year's research shows that the difficulty of parameter identification is often greater than the stability control of the system. This article is based on the above reasons, we want to reveal the role of adaptive control in the case that the system parameters can not be fully identified .This situation is very easy to happen in the practical application, so although the research of this paper is aimed at a kind of simple first order system, the result is very inspiring.

\section{Problem description}

One order system can be written as:

$$
\dot{x}=a_{1} x+a_{2} \sin x+u
$$

where $a$ is unknown constant parameter, the goal is designing a controller such that the system state $x$ can trace the expected value $x^{d}$.

\section{Design controller without adaptive control}

First, define a error variable as $z_{1}=x_{1}-x_{1}^{d}$, then

$$
\dot{z}_{1}=\dot{x}_{1}-\dot{x}_{1}^{d}=a_{1} x+a_{2} \sin x+u
$$


Design state feedback control law without using adaptive method as:

$$
u=-\sum_{i=1}^{n} k_{i} f_{i}\left(z_{1}\right)
$$

choose $n=5$,

$$
\begin{gathered}
f_{1}\left(z_{1}\right)=z_{1}, f_{2}\left(z_{1}\right)=z_{1}^{3}, f_{3}\left(z_{1}\right)=z_{1}^{1 / 3} \\
f_{4}\left(z_{1}\right)=\frac{z_{1}}{\left|z_{1}\right|+\varepsilon}, \varepsilon=0.2, \\
f_{5}\left(z_{1}\right)=\frac{1-e^{-\tau z_{1}}}{1+e^{-\tau z_{1}}}, \quad \tau=0.5
\end{gathered}
$$

where $f_{3}\left(z_{1}\right)$ is Terminal attractor, and $f_{5}\left(z_{1}\right)$ is Sigmoid function, $f_{4}\left(z_{1}\right)$ and $f_{5}\left(z_{1}\right)$ are both bounded, Obviously, $f_{i}\left(z_{1}\right)$ satisfies $z_{1} f_{i}\left(z_{1}\right) \geq 0$, then

$$
\dot{z}_{1}=a_{1} x+a_{2} \sin x-\sum_{i=1}^{n} k_{i} f_{i}\left(z_{1}\right)
$$

Choose a Lyapunov function as

$$
V=\frac{1}{2} z_{1}^{2}
$$

Solve its derivative as

$$
\dot{V}=z_{1} \dot{z}_{1}
$$

And it can be simplified as

$$
\dot{V}=z_{1} a_{1} x+z_{1} a_{2} \sin x-\sum_{i=1}^{n} k_{i} z_{1} f_{i}\left(z_{1}\right)
$$

If $\left|a_{1} x+a_{2} \sin x\right| \leq M_{a}$, then $\left|z_{1}\right| \leq M_{b}$, or

$$
\dot{V} \leq 0
$$

So according to Lyapunov theory we get $z_{1} \rightarrow 0$.

\section{Numerical simulation}

Choose unknown parameter $a_{1}=3, a_{2}=5$, initial state $x_{1}(0)=-1$, expected state $x_{1}^{d}=1$, use Simulink in Matlab, write program without improved integral adaptive identification controller, the program can be written as: 


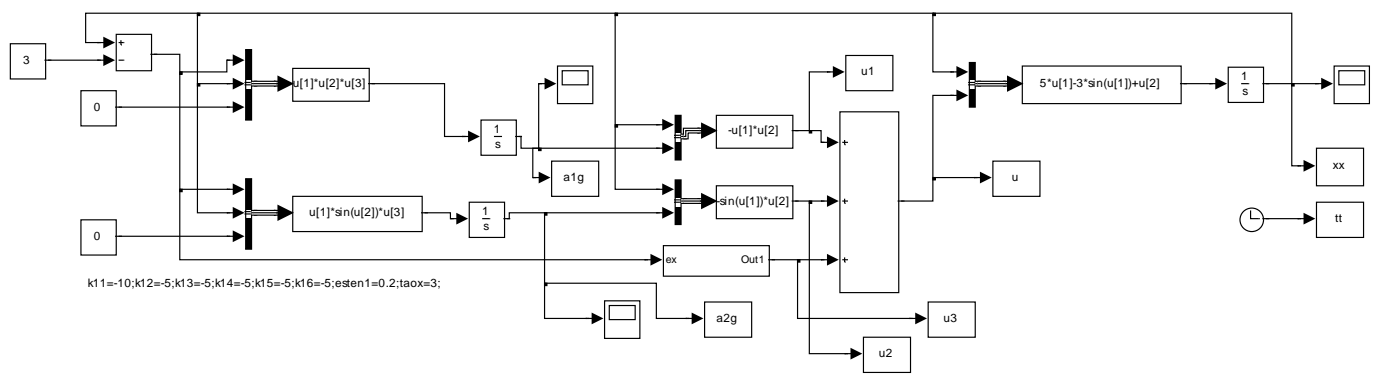

Fig.1 Program without improved integral

Choose $k_{1}=5, k_{\mathrm{s} 1}=\Gamma_{a}=1$, the simulation results are as follows:

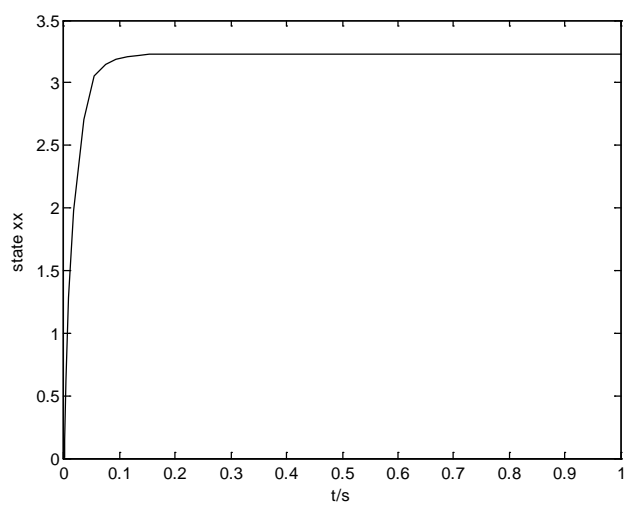

Fig.2 State $\mathrm{x}$

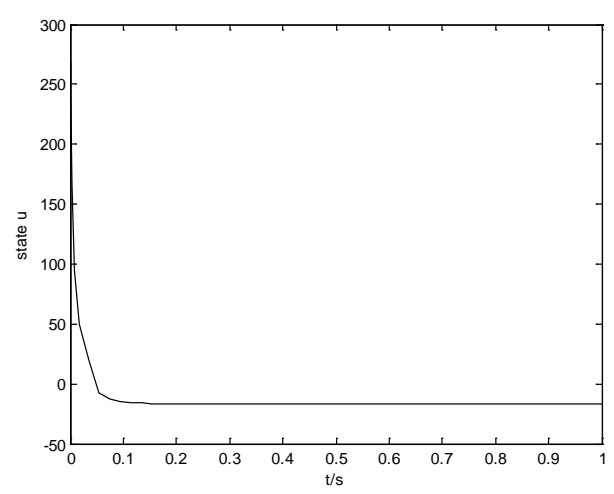

Fig.3 Control u

From the above simulation result, we can find that the system has steady state error, so a kind of integral adaptive control law can be adopt to reduce steady state error. Then the program can be revised as:

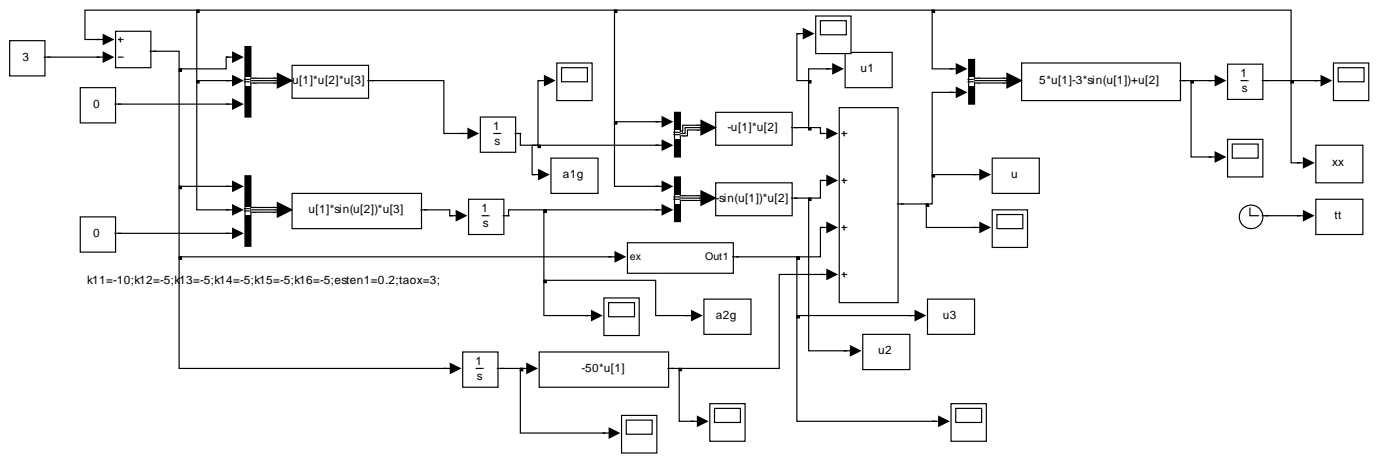

Fig.4 Program with adaptive item

Choose $k_{2}=1$, the simulation results are as follows: 


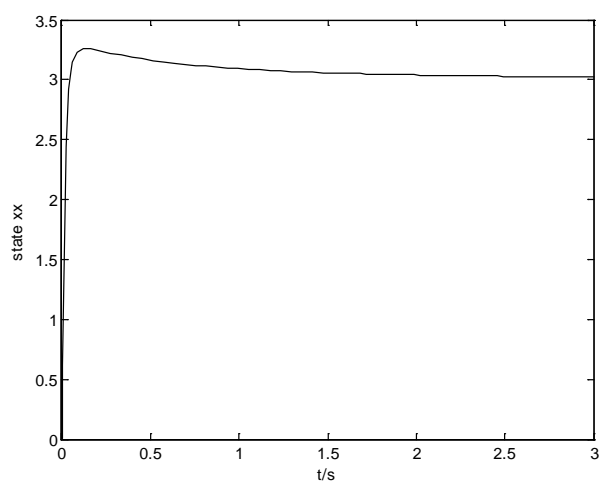

Fig.5 state a

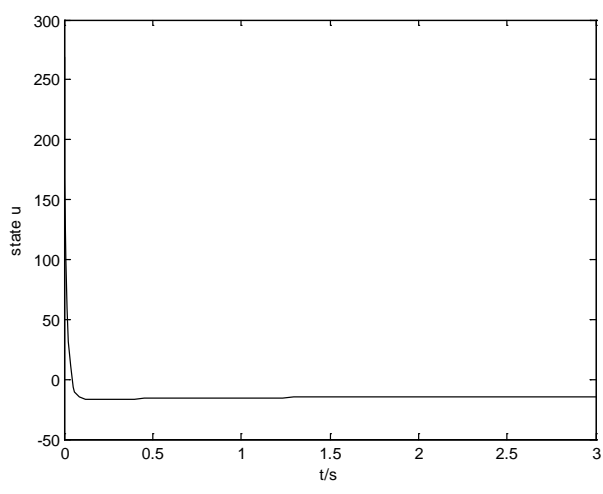

Fig.6 state $\mathrm{x} 1$

The unknown parameter can be identified. If the system don't use integral control, that is $k_{s 1}=0$, the simulation results are as follows:

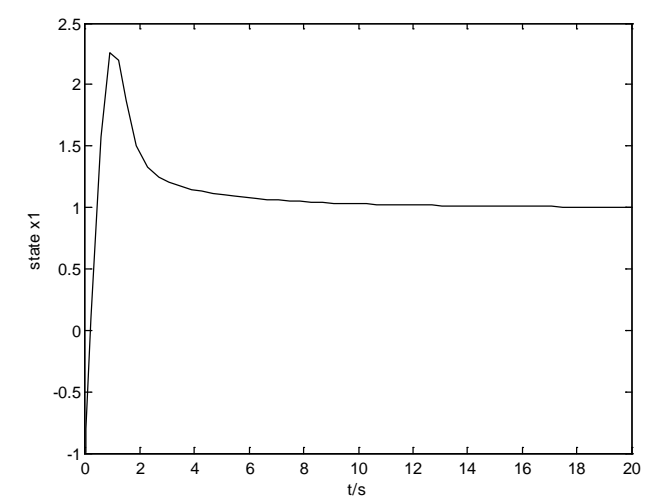

Fig.7 State $\mathrm{x}$

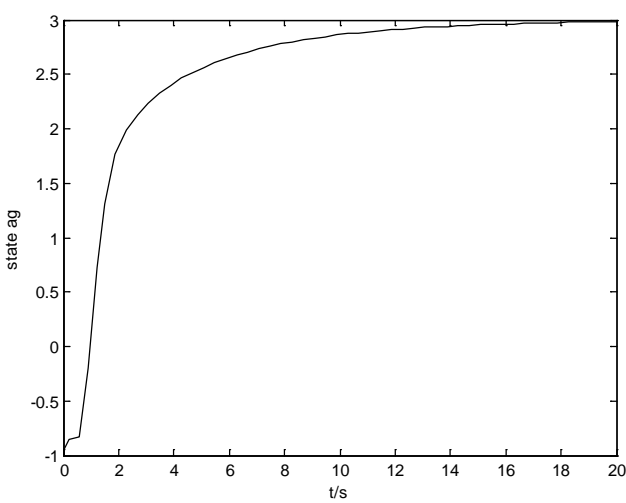

Fig.8 Control u

According to above simulation result, we can make a conclusion that the adaptive item can reduce the steady state error.

\section{Conclusion}

In this paper, the theory of adaptive control and non adaptive control for a class of simple systems with unknown parameters is analyzed, and the simulation results are compared and analyzed. The simulation results show that the adaptive control law can effectively eliminate the error of the system when the parameters are not accurately identified, and the adaptive control law can play the role of integral control.

\section{Reference}

[1] Maurizio C, Marcello P, Giansalvo, et al. A new experimental application of least-squares techniques for the estimation of the induction motor parameters[J]. IEEE Transactions on Industry Applications, 2003, 39(5):1247-1256.

[2] Raj M B, Alexander G P, Hamid A T, Neural speed filtering for sensorless induction motor drives[J]. Control Engineering Practice, 2004, 12(6): 687-706.

[3] Huang K S, Kent W, Wu Q, et al. Parameter identification of an induction machine using genetic algorithms[C] // Proceedings of the 1999 IEEE International Symposium on Computer Aided Control System Design. [S. L.]: [s. N.],1999: 510-515.

[4] Huynh D C, Dunnugan M W. Parameter estimation of an induction machine using advanced particle swarm optimisation algorithms[J]. IET Electric Power Applications, 2010, 4(9): 748-760. 
[5] Azinheira J R, Moutinho A, Paiva E C. A backstepping controller for path-tracking of an underactuated autonomous aitship.[J] International Journal of Robust and Nonlinear Control, 2009, 19(4):418-441

[6] Benjovengo F P, Paiva E C. Sliding mode control approaches for an autonomous unmanned airship. AIAA 2009-2869, 2009

[7] Park, C.S., H.Lee, M.J.Tahk, et al. Airship control using neural network augmented model inversion[J]. IEEE Conference on Control Applications. 2003

[8] Xie, S.R., J.Luo, J.J.Rao, et al. Computer Vision-based Navigation and Predefined Track Following Control of a Small Robotic Ariship. 2007.33(3):286-291.

[9] Rao, J., Z.Gong, J.Luo, et al., Robotics airship mission path-following control based on ANN and human operator's skill. 2007.29:5-15. 